\title{
Numerical Modelling of Percutaneous Auricular Vagus Nerve Stimulation: A Realistic 3D Model to Evaluate Sensitivity of Neural Activation to Electrode Position
}

\author{
Amine M. Samoudi ${ }^{* 1}$, Stefan Kampusch ${ }^{2}$, Emmeric Tanghe ${ }^{1}$, Jozsef C. Széles ${ }^{3}$, Luc Martens ${ }^{1}$, \\ Eugenijus Kaniusas $^{2}$ and Wout Joseph ${ }^{1}$
}

${ }^{1}$ Department of Information Technology Ghent University/imec, Ghent, Belgium.

${ }^{2}$ Research Group Biomedical Sensing, Institute of Electrodynamics, Microwave and Circuit Engineering, TU Wien, Vienna, Austria.

${ }^{3}$ Department of Surgery, Medical University Vienna, Vienna, Austria

${ }^{*}$ Correspondence to: Amine M. Samoudi, MS. Department of Information Technology (INTEC), Ghent University/imec, Gaston crommenlaan 8 box 201, 9050 Ghent, Belgium.

E-mail: amine.samoudi@ugent.be

The total number of words of the manuscript: 5270

The number of words of the abstract: 199

The number of figures: 7

The number of tables: 1 


\begin{abstract}
Objective: Percutaneous stimulation of the auricular branch of the vagus nerve (pVNS) by miniaturized needle electrodes in the auricle gained importance as a treatment for acute and chronic pain. The objective is to establish a realistic numerical model of pVNS and investigate the effects of stimulation waveform, electrodes' depth, and electrodes' position on nerve excitation threshold and the percentage of stimulated nerves.

Methods: Simulations were performed with Sim4Life. An electro-static solver and neural tissue models were combined for electromagnetic and neural simulation. The numerical model consisted of a realistic high resolution model of a human ear, blood vessels, nerves, and 3 needle electrodes.

Results: A novel 3D ear model was established, including blood vessels and nerves. The electric field distribution was extracted and evaluated. Maximum sensitivity to needles' depth and displacement was evaluated to be $9.8 \%$ and $15.5 \%$ per $0.1 \mathrm{~mm}$, respectively. Stimulation was most effective using bi-phasic compared to mono-phasic pulses.

Conclusion: The established model allows easy and quantitative evaluation of various stimulation setups, enabling optimization of pVNS in experimental settings. Results suggest a high sensitivity of pVNS to the electrodes' position and depth, implying the need for precise electrode positioning. Validation of the model needs to be performed.
\end{abstract}

Keywords: Auricular Branch of the Vagus Nerve, Neuromodulation, SENN, Electromagnetic Simulation 


\section{Authors' biography}

ir. Amine M. Samoudi is a PhD student in wireless, acoustics, environment and expert systems (UGent/imec). His current research involves neurostimulation, multimodality imaging, and EM exposure.

DI Stefan Kampusch is working towards his $\mathrm{PhD}$ on personalized auricular vagus nerve stimulation at TU Wien. He leads the working module Vagus Nerve Stimulation of COST Action BM1309.

Prof. Emmeric Tanghe is a Post-Doctoral Fellow of the FWO-V in radio propagation and part-time professor in medical applications of electromagnetic fields in and around the human body.

Dr. med. Jozsef C. Széles is a doctor of medicine at University Clinic for Surgery, Medical University of Vienna. He is the head of the Special Outpatient Clinic for Wound Surgery and Pain Management.

Prof. Luc Martens is a professor at UGent. His interests are in modelling and measurement of electromagnetic channels and exposure and energy consumption.

Prof. Eugenijus Kaniusas is the head of the research group Biomedical Sensing and chair of the advisory board of study affairs of Biomedical Engineering at TU Wien.

Prof. Wout Joseph is a professor in the domain of experimental characterization of wireless communication systems: UGent. He specializes in EM exposure, propagation, and wireless performance analysis. 


\section{INTRODUCTION}

Stimulation of the cervical vagus nerve by implanted stimulation devices gained importance as a treatment for therapy refractory epilepsy, major depression, and congestive heart failure $[1,2]$. To cope with the associated risks, less invasive techniques were developed. Those include the percutaneous stimulation of the auricular branch of the vagus nerve (pVNS) [3, 4]. By using needle electrodes in targeted regions of the auricle [5], one can easily access these nerve branches [6]. By this a minimally-invasive way of neuromodulative intervention is available. Current clinical applications include the treatment of chronic pain [7] or peripheral arterial disease (PAD) [8].

Specific stimulation of afferent vagus nerve fibers can activate autonomous modulation [9]. Sympathetic and parasympathetic branches of the autonomic nervous system modulate heart rate, blood pressure, or vascular tone [10]. A favorable parasympathetic/vagal stimulation may thus downregulate vascular tone [11], increase blood perfusion [8, 12], and downregulate inflammation [13, 14], all highly beneficial for instance in the treatment of PAD.

With a prevalence of $12-14 \%$ in the general population, PAD is a major health burden [15]. First clinical attempts using pVNS in patients suffering from PAD induced claudication showed remarkable effects and a significant prolongation of pain-free walking distance [8]. Effects seem to be superior to other possibilities of therapy using coordinated physical training or (expensive) vasoactive drugs.

Main shortcoming of current applications in pVNS is the unguided/empirical selection of both stimulation regions and parameters with unknown variation of therapeutic effect. However, high specificity of stimulation seems to be of huge importance due to the dense innervation of the auricle with nerve fibers of different origin [5], which all may react differently to stimulation and can elicit opposite physiological reactions [16].

Simulations of electromagnetic fields excited in human biological tissue by artificial neurostimulation exist in literature. However, these studies are limited to the most common types of neurostimulation, e.g., for Deep Brain Stimulation [17, 18], Spinal Cord Stimulation [19], Peripheral Nerve Stimulation [20], and Transcranial Magnetic Stimulation $[21,22]$. These studies provide us with a better understanding of the electric potential and current distributions in the biological tissues surrounding the stimulation electrodes. Some studies assume a simplified neural 
network that distributes the axons uniformly around the stimulation electrode with one and the same direction for all axons, see e.g., [23]. Using the potential distribution, it is then possible to calculate which axons are effectively stimulated. However, neural stimulation also heavily depends on the orientation of the externally applied electric field with respect to the orientation of the axon. Therefore, an improvement is to use the actual, often winding geometry of nerve axons in the simulation, as given in e.g., [17, 24].

The objective and novelty of this study is to establish, for the first time, a realistic model of the pVNS application and investigate the effect of the electrodes' depth and position, as well as the stimulation pattern on the excitation threshold in single and bundled axons. With this, the feasibility of the used model and tools for the given application is evaluated. In combination with future experimental and clinical evaluations, this should enable the development of simulation guided pVNS with better specificity and parameter selection.

\section{MATERIALS AND METHODS}

\section{A. Simulation platform}

Numerical simulations of pVNS were performed in Sim4Life [25]. We used the low frequency solver for the electromagnetic (EM) simulation. The magnetic field is neglected and the electric field is calculated only in the lossy domain (region where conductivity $\neq 0 \mathrm{~S} / \mathrm{m}$ ) covered by the default grid. The default grid provides a first gridding of the model that the user can optimize. The gridding was calculated using the stationary currents model of Sim4Life. The model was meshed approximately to 4.105 Mcells. Boundary conditions are set using Neumann conditions with a constant flux equal to zero (the derivative of a solution is set to zero on the boundary of the domain) to simulate an infinite system. The boundary settings of the electrodes are set using Dirichlet conditions (value of the solution is set to a constant value on the boundary of the domain) with a constant potential. This leads to electric fields and current densities equal to zero outside the simulation domain. This model can be used in electro quasi-static simulations when the ohmic current dominates the displacement current.

We performed all neuronal simulations using the neuronal tissue models from Sim4Life. The models (TNEURO) enable the dynamic modeling of EM-induced neuronal activation and inhibition using either complex, multi-compartmental representations of axons, nerves as bundles of axons, and neuronal networks with varying 
channel dynamics, or generic models. The neuronal tissue models from Sim4Life were validated and used to investigate electromagnetic field interactions with nerves in complex tissue-structure environments [26, 27].

Sim4Life uses the NEURON solver (Yale University, CT, USA) to simulate the effect of electromagnetic fields on neuronal dynamics. It offers the possibility to directly couple the results of EM simulations with the neuronal dynamics solver. The NEURON solver offers the possibility of an external modulating pulse $a(t)$, which can be superimposed on the static current calculated from the current density sensed by the axon exposed to an EM filed.

We used the titration mechanism to calculate the minimum amplitude of the current pulse needed to depolarize the axons. Titration is the process of stimulating an axon with a series of pulses of increasing (or varying) intensity to find the threshold above which a single action potential is generated, introducing an additional scaling factor that is varied until a response is detected. The final threshold current is the product of the static current from the initial EM field $I$, the modulating pulse $a(t)$, and the titration factor $T: I_{T}(t)=I^{*} T^{*} a(t)$. The fact that electrostatic equations are linearly separable into linear temporal components and non-linear spatial components makes the titration approach valid and suitable for the determination of axons' activation thresholds." We used the spatially extended nonlinear node (SENN) $[28,29]$ model for transmembrane mechanisms to simulate the timeresponse of an axonal membrane to external injected currents.

\section{B. $\quad$ Numerical model}

The system under investigation consisted of a realistic high resolution model of a human ear (spatial resolution $3 \mathrm{~mm}$ ), 3 electrodes, and a spatial model of major blood vessels and nerves. Locations of the electrodes are based on positions used in clinical practice and on the innervation of the auricular branch of the vagus nerve given in [5]. One electrode is considered as a reference (zero potential), while the two other electrodes are considered as stimulating electrodes. Figure 1a shows a schematic overview of the numerical model. The conductivity of the vessels was set to $0.7 \mathrm{~S} / \mathrm{m}$ [25] and the ear conductivity to $0.2 \mathrm{~S} / \mathrm{m}$ based on a recent work of Santis et al. [30]. The boundary settings of the electrodes were set using Dirichlet conditions with a constant potential.

Figure $1 \mathrm{~b}$ shows locations of the vessels while Figure 1c shows locations and shapes of the nerves $(\mathrm{N})$ and axons (A) inside the ear model. Nerve1 (N1), nerve2 (N2), and nerve4 (N4) are branching nerves, while axon3 (A3) and axon5 (A5) are non-branching single axons. Locations of the vessels and nerves/axons are based on the vascularization of the auricle and the nerve supply of the human auricle studied in $[5,31,32]$. In fact, distribution of 
nerves and vessels in the auricle is highly individual, thus a typical distribution was selected. Threshold for depolarization, temperature, initial potential, axon diameter, and the nodal gap were set to $80 \mathrm{mV}, 34{ }^{\circ} \mathrm{C},-70 \mathrm{mV}$, $10 \mu \mathrm{m}$, and $2.5 \mu \mathrm{m}$, respectively [33]. The axon diameter at node $d$ and the internodal distance $L$ are related to the fiber diameter $D$ by the following formulae [28]: $d=0.7 D$ and $L=100 D$. Simulations were carried out with realistic stimulation pulses [4]. Nerves were stimulated by two types of pulses: anodic and cathodic single cycle mono-phasic (amplitude $=1 \mathrm{~V}$, duration $1 \mathrm{~ms}$ ) and bi-phasic (amplitude $= \pm 1 \mathrm{~V}$, duration $1 \mathrm{~ms}$ ) pulses (Figure 2).

For axon population simulations, 20 axons were defined from a rectangular seed region to form N1, N2, and N4; approximately $0.3 \times 0.3 \mathrm{~mm}$ in size to generate a dense population of 60 axons in total (see Figure 1d). This number of axons is based on the work of Safi et al [34] who counted the numbers of thick myelinated $A \beta$-axons in the auricular branch of the vagus nerve. Vessels and nerves are just below the epidermis layer of the skin. The epidermis thickness is about $74.9 \mu \mathrm{m}$ at the thinnest parts of the body (ear's skin, eyelids) [35]. We then used a depth of $0.07 \mathrm{~mm}$ for the nerves/vessels inside the ear. Point sensors for depolarization were placed each $1 \mathrm{~mm}$ for all the nerves/axons with respect to the used internodal distance of $1 \mathrm{~mm}$.

\section{Effect Of The Electrodes' Position And Depth On The Percentage Of Activated Axons}

To investigate the effect of the needle penetration depth on the percentage of activated axons, we changed the needle penetration depth of electrode1 (E1) and electrode2 (E2) inside the ear from $1.5 \mathrm{~mm}$ to $0.8 \mathrm{~mm}$ in steps of $0.1 \mathrm{~mm}$ (see Figure 3). The lateral position of the electrodes was also changed from the original position by $\pm 0.1 \mathrm{~mm}$ in the $\mathrm{y}$ and $\mathrm{z}$ directions, with a maximum of $( \pm 0.1, \pm 0.1) \mathrm{mm}$. Here the needle penetration was kept at the maximum value, i.e., the insulation layer was always in contact with the ear. 


\section{RESULTS}

\section{A. $\quad$ Electric field distribution}

Figure 4 shows the electric field distribution. The E-field is directed from the reference electrode (at $0 \mathrm{~V}$ ) to the cathodic electrode (E1 at $-1 \mathrm{~V})$ with maximum values near the electrodes, and is penetrating inside the ear, vessels and nerves.

\section{B. $\quad$ Stimulation thresholds for different stimulation patterns}

Figure 5 shows the spatial distribution of E-field and its $1^{\text {st }}$ derivative along N1, N2, and N4 (the activated nerves). The horizontal axis indicates the longitudinal distance along the nerve's branch triggering the first action potential. . This figure applies to a cathodic monopolar stimulus electrode (E1 for N1, E2 for N2 and N4). For the anodic monopolar electrode stimulus, the diagram in Figure 5 would be flipped about the horizontal axis.

Table 1 lists the required pulse amplitude to activate the nerves/axons as a function of the stimulation pattern. The voltage for each nerve/axon is related to the anodic mono-phasic voltage. For an amplitude of 1 V, N1, N2 and N4 will be activated (generation and propagation of the action potential, threshold in Table $1<1 \mathrm{~V}$ ), while A3 and A5 require amplitudes of $14 \mathrm{~V}$ and $3.5 \mathrm{~V}$, to be activated, respectively.

Table 1 also shows that cathodic stimulation (indicated by a minus sign) requires in general less amplitude compared to the anodic stimulation for $\mathrm{N} 1$ and $\mathrm{N} 2$ (cathodic mono-phasic pulse requires $-34 \%$ and $-50 \%$ amplitude than the anodic mono-phasic for N1 and N2, respectively), while the anodic mono-phasic pulse is the pulse requiring the least amplitude for N4. Bi-phasic pulses tend to require less amplitude to activate the nerves compared to the mono-phasic pulses. Branching nerves (N1, N2, and N4) are considered activated when a single axon of the nerve is activated and the generated action potential is propagated to all the branches of the nerves (measured by extracting action potential and titration factor in all the nodes of the nerve's branches) [33]

\section{Map of percentage of stimulated axons}

Figure 1.d shows an illustrative example of axon activation of N1 generated by an anodic mono-phasic pulse (amplitude $=1 \mathrm{~V}$, period $=1 \mathrm{~ms}$ ), activating $70 \%$ of the axons for the anodic mono-phasic pulse. Stimulation with a 
bi-phasic pulse (amplitude $=1 \mathrm{~V}$, period $=1 \mathrm{~ms}$ ) results in a $100 \%$ axon activation in $\mathrm{N} 1$. Results also show that a $9 \%$ higher amplitude is required for the anodic mono-phasic pulse to obtain $100 \%$ axon population activation of N1 than for the bi-phasic pulse.

The axon population of N4 was 100\% activated for both stimulating pulses. $95 \%$ and $100 \%$ axons of N2 were activated when stimulated by the anodic mono-phasic and the anodic bi-phasic pulses, respectively. Results also show that $2 \%$ more amplitude is needed for the anodic mono-phasic pulse to obtain $100 \%$ axon population activation of $\mathrm{N} 2$.

The axon population of N1 needs slightly higher amplitudes to be $100 \%$ activated due to the high sensitivity of the percentage of stimulated axons to the electrode position, which will be detailed in the section III.D.

\section{Effect of the electrode depth on the percentage of stimulated axons}

Figure 6 shows the percentage of the activated axons and the value needed to obtain $100 \%$ activation for axon population in $\mathrm{N} 1, \mathrm{~N} 2$, and $\mathrm{N} 4$ as a function of the electrode depth $(d)$ from $1.5 \mathrm{~mm}$ to $0.8 \mathrm{~mm}$ for anodic monophasic and bi-phasic pulses. The voltage for each nerve is related to the value at $1.5 \mathrm{~mm}$ (initial depth of the electrode) for each nerve.

First observations highlight that the axon population of N4 is the most activated among the three sets of nerves and the bi-phasic pulses require less amplitude to activate the axon population. These observations are in coherence with results for single axons.

The axon population of N1 was the most sensitive to the electrode depth (maximum sensitivity of $9.81 \%$ per $0.1 \mathrm{~mm}$ for the $0.8 \mathrm{~mm}$ depth in Figure 6). Results also show that $\% \%$ axon activation is obtained for depths less than $1 \mathrm{~mm}$ for all the nerves and all the pulses.

\section{E. Effect of the electrode position on the percentage of stimulated axons}

Figure 7 shows the percentage of the activated axons and the value needed to obtain $100 \%$ activation for the axon population of $\mathrm{N} 1$ as a function of the electrode 1 displacement with $\pm 0.1 \mathrm{~mm}$ in the $\mathrm{y}$ and $\mathrm{z}$ directions (y, z), as shown in Figure 3 for some displacements.

Results show that the percentage of activated axons is sensitive to the position of the electrode with a maximum sensitivity of $15.5 \%$ for each $0.1 \mathrm{~mm}$ (positions: $(-0.1,0.1)$ and $(0.1,0.1))$. We also remark that some 
positions provide a higher percentage of activated axons than the original position $((-0.1,-0.1),(0,-0.1)$, and $(0.1,-$ 0.1 ), Figure 3).

\section{DISCUSSION}

A realistic numerical model of the pVNS, for the first time, has been developed and investigated. Electric field distribution shows that higher electric field values appear near the needle, which is due to the point effect on the needle.

Results show that A3 and A5 were not activated for the amplitude of $1 \mathrm{~V}$. These axons are on the back of the ear and thus less exposed to the electric field generated by the electrodes positioned in the front of the ear. Stimulation was most effective using cathodic pulses than anodic pulses for N1 and N2 while the inverse is true for N4. To be able to excite an axon, the membrane should be adequately depolarized. Maximum depolarization occurs where the spatial gradient of the E-field is maximally positive [33]. The spatial gradient of the internal E-field reaches higher maximum values for the cathodic stimulation compared to the anodic stimulation for N1 and N2 (cathodic: $\approx 0.2$ of the maximum absolute value of the N4' 1st derivative for N1 and N2. anodic: 0.16 and 0.1 of the maximum absolute value of the N4' 1st derivative for N1 and N2, respectively), and the inverse is true for the N4 ( 0.74 and 1 for cathodic and anodic, respectively). This difference between anodic and cathodic stimulation is less important for the bi-phasic pulses. Concerning the most effective stimulation pattern, the bi-phasic pulses tend to require less amplitude to activate the nerves/axons compared to the mono-phasic pulses. This can be explained by the fact that the bi-phasic pulses combine anodic and cathodic mono-phasic pulses, thus there is an additional chance for stimulation at the phase reversal, especially with pulse durations longer than $0.5 \mathrm{~ms}$. Results of Table 1 show also that the stimulation thresholds for the bi-phasic pulses are almost equal to the lowest threshold of the anodic and the cathodic mono-phasic pulses for each nerve/axon [33].

N1 requires the least amplitude for the anodic pulses, while N2 requires the least amplitude for the cathodic pulses (N1: $0.77 \mathrm{~V}$ and $0.51 \mathrm{~V}$ for anodic and cathodic mono-phasic pulse, respectively. N2: $0.88 \mathrm{~V}$ and $0.44 \mathrm{~V}$ for anodic and cathodic mono-phasic pulse, respectively, Table 1). This is explained in Figure $5 \mathrm{~b}$ where the $1^{\text {st }}$ derivative of E-field has a slightly higher maximum positive value for N2 compared to N1 for the cathodic stimulation $(2.22 \%$ 
higher for $\mathrm{N} 2$ compared to N1), and higher maximum positive value for N1 compared to N2 for the anodic stimulation.

N4 requires the least amplitude for the stimulation among all the nerves, which is explained by the highest value of the maximum positive value of the $1^{\text {st }}$ derivative of E-field for the anodic stimulation (maximum $1^{\text {st }}$ derivative of E-field for N4 is 5 times higher than those of N1 and N2 in Figure 5b).

The axon population of $\mathrm{N} 1$ was the most sensitive to the electrode depth with no axon activation registered for needle depths less than $1 \mathrm{~mm}$ for all the pulses (Figure 6 and Figure 7). This is due to the weak inner electric field, in case that the needle does not penetrate the ear to a sufficient extent. This percentage can be changed to 100 $\%$ if we increase the initial amplitude by $68.7 \%$ at least $(1.84 \mathrm{~V})$. Results show the importance of the electrode penetration depth for nerve/axon activation. At least $1 \mathrm{~mm}$ penetration depth should be kept to ensure complete nerve activation with a value of $1.6 \mathrm{~V}$ pulse amplitude.

Some electrode positions provide a higher percentage of activated axons than the original position, which can be used to further decrease the pulse amplitude (Figure 7). At these positions, the electrode needles are slightly closer to the axon population, exposing the axons to higher values of electric field. Sensitivity results show that the stimulation thresholds and the percentage of activated axons are sensitive to the electrodes' position. Taking into account the specificity of each patient's nerves and ear anatomy, special attention should be paid to the placement of the electrodes for each specific patient.

The feasibility and plausibility of the given model and tools is demonstrated. However, the robustness of the model has to be checked in future works. Results of simulations provide an impression in the field distribution and thus stress the necessary accuracy while placing electrodes. The model may explain physiological findings in regions known to be innervated by more than one nerve. Furthermore, the model may allow personalization of stimulation when supported by experimental and clinical data. As the physiology of each patient is different to a degree, a typical distribution of the nerves and vessels was selected. The proposed work does not consider the effect of connection between the axons; all the axons in the model are considered separately. Electrode-interface effects were not modelled in this study and we used constant voltage simulation. Initially, voltages applied to an electrode will result 
in nerves being exposed to EM fields based on the configuration of the electrode voltage. The nerves will sense an electric potential distribution that can be derived from the E-field. By applying an external modulating pulse, the external potential sensed by the nerve will then be modulated and scaled by the desired pulse (mon-phasic, biphasic). These limitations could lead to some remnant errors. Nevertheless, it is possible to gain useful information for the necessity of accuracy when placing electrodes by evaluating simple nerve structures.

\section{CONCLUSION}

A realistic numerical model for pVNS application at the human auricle including vessels and nerves was developed. The numerical model was used to investigate the percentage of the activated axons with respect to stimulation waveform, electrode depth, and electrode position. Results show that a cathodic bi-phasic pulse, besides

being charge balanced, is the pulse requiring the least amplitude to activate the nerves. Results also show quantitative sensitivity of the stimulation thresholds to the electrodes' position and depth, which can affect the important specificity of pVNS. Thus special attention should be paid to the placement of the electrodes for each specific patient. The proposed model does not consider any network between the nerves, and relies on the SENN model for transmembrane mechanisms and axon activation. Future works will further evaluate the model to investigate additional stimulation patterns (tri-phasic pulses, pulses with equal energy) and burst stimulation as well as the robustness of the model. Experimental pVNS validation of the set-ups from numerical modelling and mutual optimization of the model and the experimental setup will also be performed using clinical studies.

\section{ACKNOWLEDGMENTS}

The research was supported by COST Action BM1309 (COST EMF-MED) and the FWO G003415N project.

E. Tanghe, is a post-doctoral fellow of the Research Foundation-Flanders (FWO-V).

\section{REFERENCES}

[1] Groves, D. and Brown, V. (2005). Vagal nerve stimulation: a review of its applications and potential mechanisms that mediate its clinical effects. Neuroscience and Biobehavioral Reviews, 29(3):493-500. 
[2] De Ferrari, G., Crijns, H., Borggrefe, M. et al. (2011). Chronic vagus nerve stimulation: a new and promising therapeutic approach for chronic heart failure. European Heart Journal, 32(7):847-855.

[3] Ellrich, J. (2011). Transcutaneous Vagus Nerve Stimulation. European Neurological Review, 6(4), 254.

[4] Kampusch, S., Kaniusas, E. and Széles, J. (2013). New Approaches in Multi-Punctual Percutaneous Stimulation of the Auricular Vagus Nerve. Proceedings of the 6th International IEEE EMBS Conference on Neural Engineering 263-266.

[5] Peuker, E. and Filler, T. (2002). The nerve supply of the human auricle. Clin. Anat., 15(1):35-37.

[6] Kaniusas, E., Varoneckas, G., Mahr, B., and Széles, J. (2011). Optic Visualization of Auricular Nerves and Blood Vessels: Optimisation and Validation. IEEE Transactions On Instrumentation And Measurement, 60(10):3253-3258.

[7] Sator-Katzenschlager, S., Széles, J., Scharbert, G., Michalek-Sauberer, A., Kober, A., Heinze, G., and KozekLangenecker, S. (2003). Electrical Stimulation of Auricular Acupuncture Points Is More Effective Than Conventional Manual Auricular Acupuncture in Chronic Cervical Pain: A Pilot Study. Anesthesia and Analgesia, 1469-1473.

[8] Payrits, T. et al (2011). Vagal stimulation - a new possibility for conservative treatment of peripheral arterial occlusion disease. Zentralblatt für Chirurgie 136:431-35.

[9] Berthoud, H. and Neuhuber, W. (2000). Functional and chemical anatomy of the afferent vagal system. Autonomic Neuroscience, 85(1-3):1-17.

[10] Kaniusas, E. (2012). Biomedical Signals and Sensors I. Published by Springer, 2012.

[11] Zamotrinsky, A., Kondratiev, B., and de Jong, J. (2001). Vagal neurostimulation in patients with coronary artery disease. Autonomic Neuroscience, 88(1-2):109-116.

[12] Széles, J. and Litscher, G. (2004). Objectivation of cerebral effects with a new continuous electrical auricular stimulation technique for pain management. Neurological Research, 26(7):797-800.

[13] Tracey, K. (2009). Reflex control of immunity. Nature Reviews Immunology, 9(6):418-428.

[14] Zhao, Y., He, W., Jing, X., Liu, J., Rong, P., and Ben, H. et al. (2012). Transcutaneous Auricular Vagus Nerve Stimulation Protects Endotoxemic Rat from Lipopolysaccharide-Induced Inflammation. Evidence-Based Complementary And Alternative Medicine, 2012:1-10.

[15] Shammas, N. (2007). Epidemiology, classification, and modifiable risk factors of peripheral arterial disease. Vascular Health And Risk Management, 3(2):229-234.

[16] Gao, X., Zhang, S., Zhu, B., and Zhang, H. (2008). Investigation of specificity of auricular acupuncture points in regulation of autonomic function in anesthetized rats. Autonomic Neuroscience, 138(1-2):50-56. 
[17] Miocinovic, S. (2006). Computational Analysis of Subthalamic Nucleus and Lenticular Fasciculus Activation During Therapeutic Deep Brain Stimulation. Journal Of Neurophysiology, 96(3):1569-1580.

[18] Schmidt, C. and van Rienen, U. (2012). Modeling the Field Distribution in Deep Brain Stimulation: The Influence of Anisotropy of Brain Tissue. IEEE Transactions On Biomedical Engineering, 59(6):1583-1592.

[19] Danner, S.M. et al. (2014). Potential distribution and nerve fiber responses in transcutaneous lumbosacral spinal cord stimulation. Int. Conference on Advancements of Medicine and Health Care through Technology; IFBME 44, 203-208.

[20] Samoudi, A., Vermeeren, G., Tanghe, E., Van Holen, R., Martens, L., and Josephs, W. (2016). Numerically simulated exposure of children and adults to pulsed gradient fields in MRI. J. Magn. Reson. Imaging, 44: 1360-1367

[21] De Lucia, M., Parker, G., Embleton, K., Newton, J., and Walsh, V. (2007). Diffusion tensor MRI-based estimation of the influence of brain tissue anisotropy on the effects of transcranial magnetic stimulation. Neuroimage, 36(4):1159-1170.

[22] Lu, M. and Ueno, S. (2013). Calculating the induced electromagnetic fields in real human head by deep transcranial magnetic stimulation. Conf. Proc. IEEE Eng. Med. Biol. Soc. 2013:795-8.

[23] Choi, C., Lee, Y. and Tsou, Y. (2011). Modeling Deep Brain Stimulation Based on Current Steering Scheme. IEEE Transactions on Magnetics, 47(5):890-893.

[24] Chaturvedi, A., Foutz, T. and McIntyre, C. (2012). Current steering to activate targeted neural pathways during deep brain stimulation of the subthalamic region. Brain Stimulation, 5(3):369-377.

[25] Sim4Life, Zurich Med Tech, www.zurichmedtech.com/sim4life/, visited on September 2016.

[26] Neufeld, E., Cassará, A., Montanaro, H., Kuster, N. and Kainz, W. (2016). Functionalized anatomical models for EMneuron Interaction modeling. Physics in Medicine and Biology, 61(12):4390-4401.

[27] Neufeld, E., Vogiatzis Oikonomidis, I., Ida Iacono, M., Angelone, L., Kainz, W. and Kuster, N. (2016). Investigation of assumptions underlying current safety guidelines on EM-induced nerve stimulation. Physics in Medicine and Biology, 61(12):4466-4478.

[28] Reilly, J., Freeman, V. and Larkin, W. (1985). Sensory Effects of Transient Electrical Stimulation - Evaluation with a Neuroelectric Model. IEEE Transactions on Biomedical Engineering, BME-32(12):1001-1011.

[29] McNeal, D. (1976). Analysis of a Model for Excitation of Myelinated Nerve. IEEE Transactions on Biomedical Engineering, BME-23(4):329-337.

[30] Santis, V., Chen, X., Laakso, I. and Hirata, A. (2015). An equivalent skin conductivity model for low-frequency magnetic field dosimetry. Biomedical Physics \& Engineering Express, 1(1). 
[31] Tilotta, F., Lazaroo, B., Laujac, M. and Gaudy, J. (2009). A study of the vascularization of the auricle by dissection and diaphanization. Surgical and Radiologic Anatomy, 31(4):259-265.

[32] Alvord, L. and Farmer,B. (1997). Anatomy and orientation of the human external ear. J. Am. Acad. Audiol., 8(6):38390.

[33] Reilly, J. and Diamant, A. (2011). Electrostimulation-Theory Applications, and Computational Model. Published by Boston: Artech House.

[34] Safi, S., Ellrich, J. and Neuhuber, W. (2016). Myelinated Axons in the Auricular Branch of the Human Vagus Nerve. Anat. Rec., 299(9):1184-1191.

[35] Sandby-Møller, J., Poulsen, T. and Wulf, H. (2003). Epidermal Thickness at Different Body Sites: Relationship to Age, Gender, Pigmentation, Blood Content, Skin Type and Smoking Habits. Acta Dermato-Venereologica, 83(6):410-413. 


\section{Tables}

TABLE 1

PERCENTAGE OF THE STIMULATION THRESHOLDS RELATED TO THE ANODIC MONO-PHASIC VOLTAGE OF EACH

NERVE/AXON

Nerve/Axons

Pulse type Threshold amplitude (V)

\begin{tabular}{ccc}
\hline N1 & Mono-phasic + & 0.77 \\
& Bi-phasic + & $-36 \%$ \\
\hline & Mono-phasic- & $-34 \%$ \\
\hline N2 & Bi-phasic- & $-35 \%$ \\
\hline & Mono-phasic + & 0.88 \\
\hline & Bi-phasic + & $-53 \%$ \\
\hline & Mono-phasic- & $-50 \%$ \\
\hline & Bi-phasic- & $-52 \%$ \\
\hline
\end{tabular}

\begin{tabular}{ccc}
\hline \hline A3 & Mono-phasic + & 14.0 \\
& Bi-phasic + & $-57 \%$ \\
\hline & Mono-phasic- & $-57 \%$
\end{tabular}

Bi-phasic- $\quad-56 \%$

\begin{tabular}{ccc}
\hline \hline N4 & Mono-phasic + & 0.22 \\
\hline & Bi-phasic + & $0 \%$ \\
& Mono-phasic- & $+18 \%$ \\
\hline B5 & Bi-phasic- & $-4 \%$ \\
\hline \hline & Mono-phasic + & 3.5 \\
\hline & Bi-phasic + & $+8 \%$ \\
\hline & Mono-phasic- & $+20 \%$ \\
\hline \hline & Bi-phasic- & $-2 \%$ \\
\hline +refers to the anodic pulse, while - refers to the cathodic pulse.
\end{tabular}

The voltage for each nerve/axon is related (in percentage) to the anodic mono-phasic voltage of each nerve/axon. 


\section{Figure Legends}

Figure 1: Numerical model of the pVNS application. (a) Scheme of the application. (b) Auricle, vessels, nerves, and the electrodes. Vessels and nerves are inside the ear and not on the surface of the ear. (c) Locations and shapes of the nerves. (d) Activation of the axon population of N1 stimulated by anodic mono-phasic pulses. Dark color indicates inactivated axons.

Figure 2: Mono-phasic and bi-phasic stimulation pulses.

Figure 3: Scheme of the change in penetration depth and position with respect to the axon bundle

Figure 4: Electric field distribution, in $\mathrm{dB}$ normalized to $500 \mathrm{~V} / \mathrm{m}$. Red electrode has a potential of $-1 \mathrm{~V}$. The blue electrode is the reference $(0 \mathrm{~V})$.

Figure 5: (a) Spatial distribution of E-field and its $1^{\text {st }}$ derivative along N1, N2, and N4 (the activated nerves). The $1^{\text {st }}$ derivative was normalized to the maximum absolute value of the $\mathrm{N} 4{ }^{\prime} 1^{\text {st }}$ derivative.

Figure 6: Percentage of activated axons of N1, N2, and N4 and increase in the amplitude needed to activate $100 \%$ of the axon population around each nerve, related to the value needed for $100 \%$ axon activation for the $1.5 \mathrm{~mm}$ depth position for different electrode depths (d). * Related to the value needed for $100 \%$ axon activation for the $1.5 \mathrm{~mm}$ depth position.

Figure 7: Percentage of axon activation and the amplitude needed for $100 \%$ axon activation for different positions of elctrode1. These values are for axons of N1 stimulated by the anodic mono-phasic pulse. 

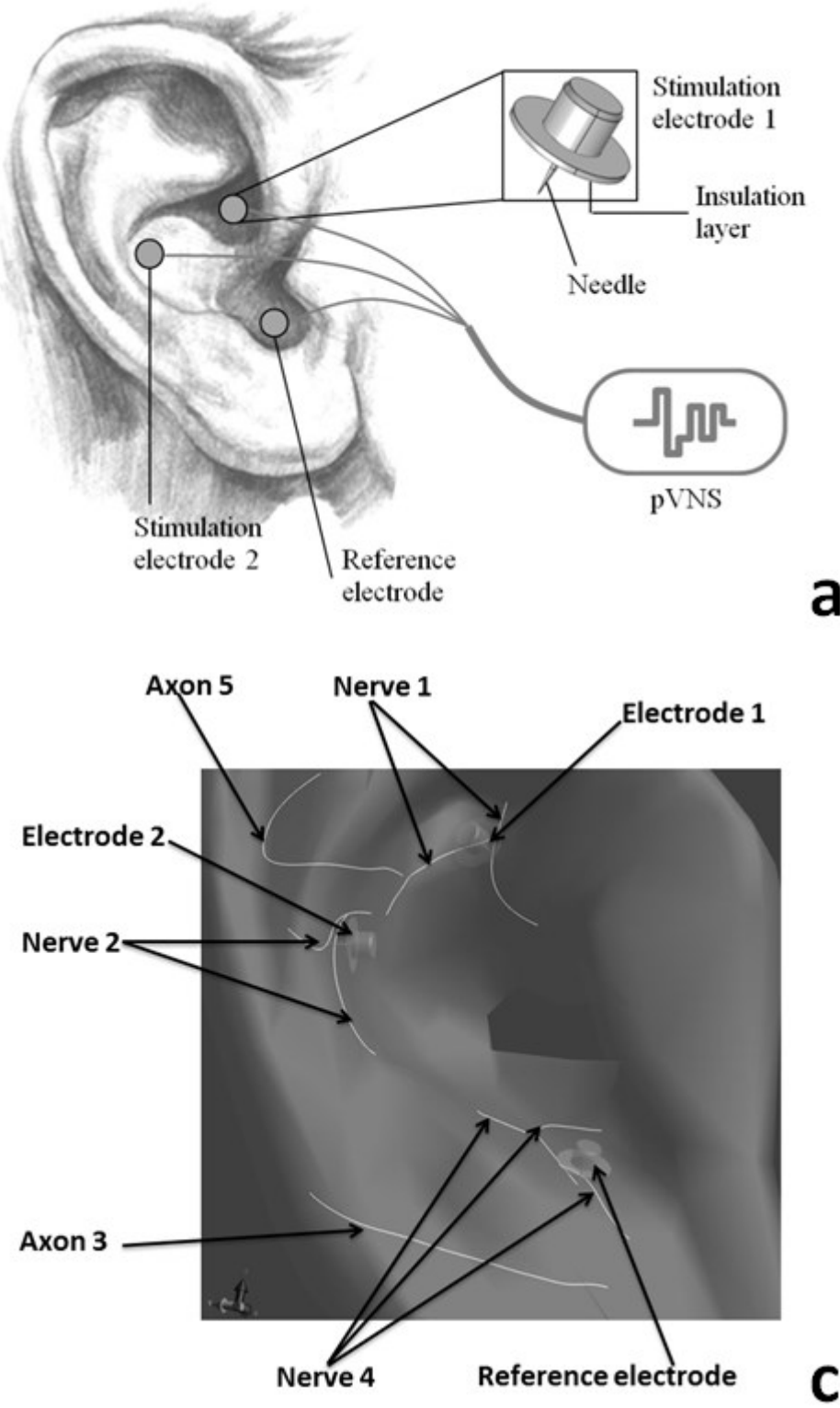

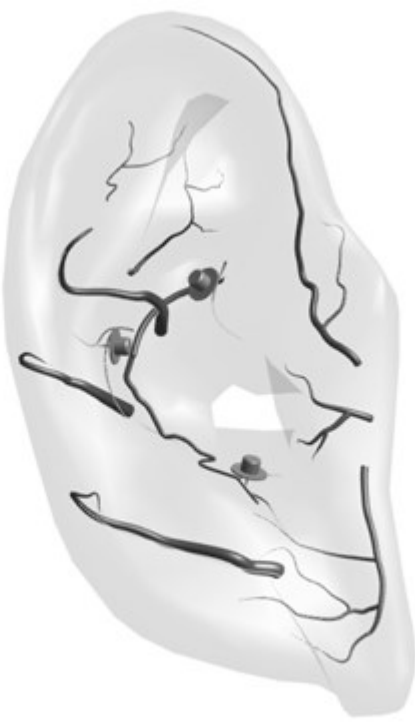

a

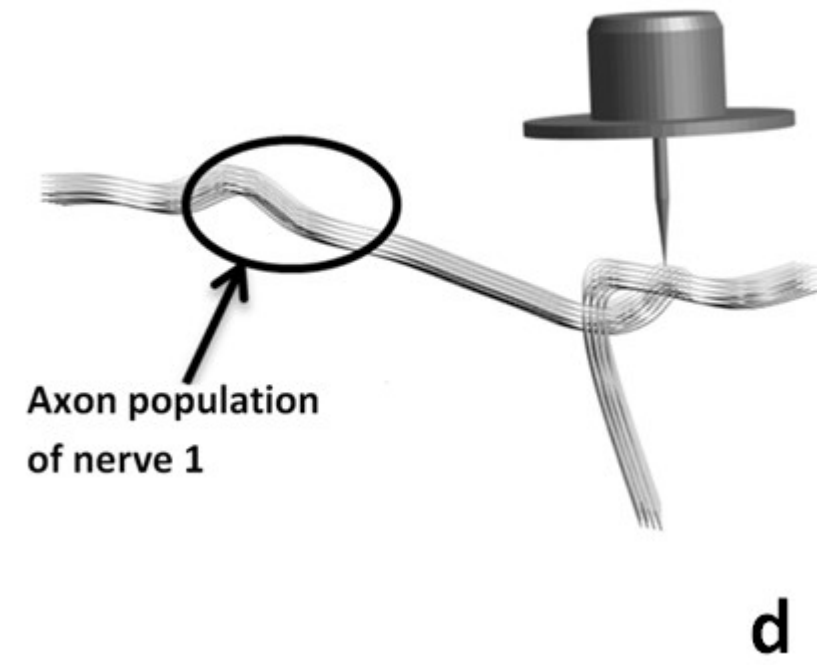

Figure 1 


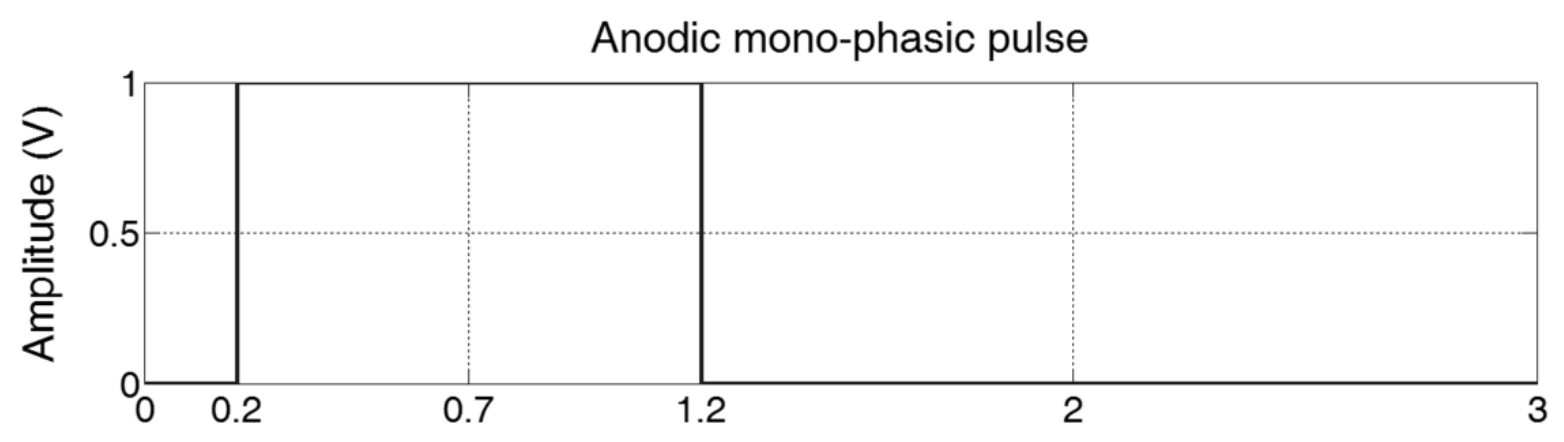

Anodic bi-phasic pulse

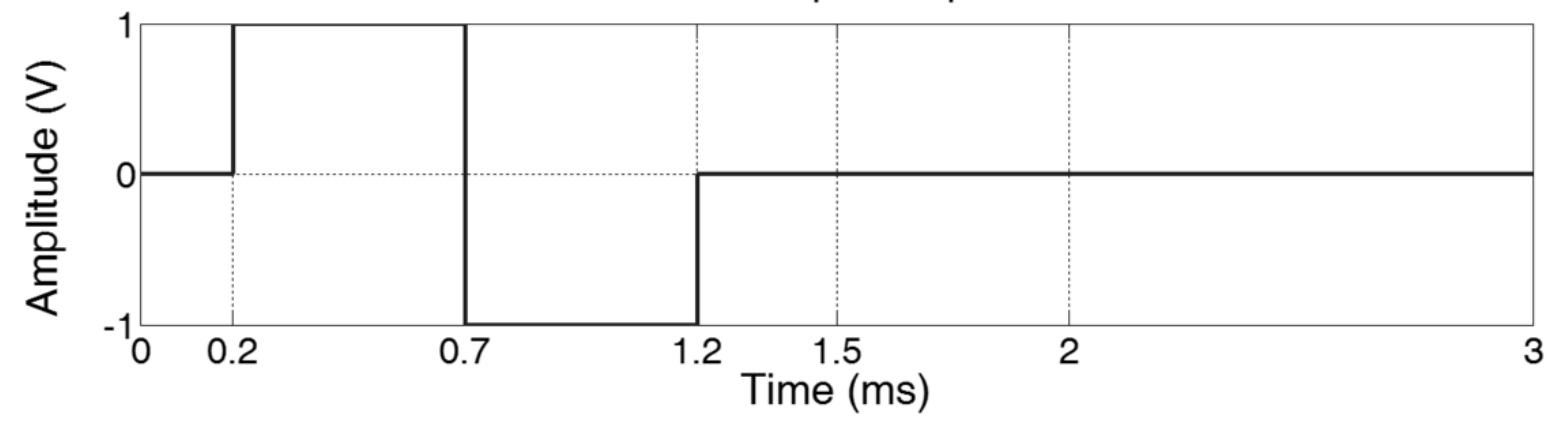

Figure 2 


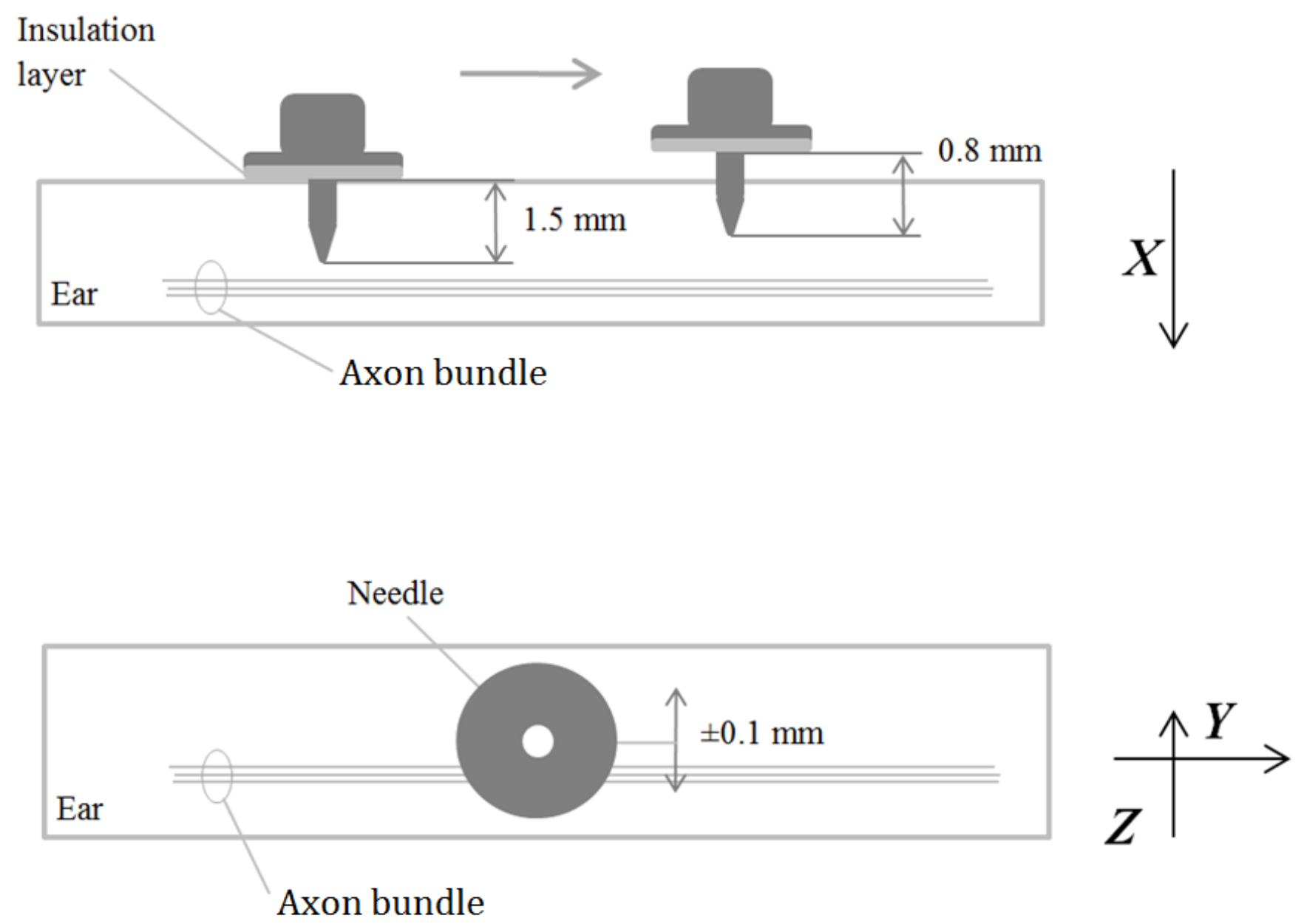

Figure 3 


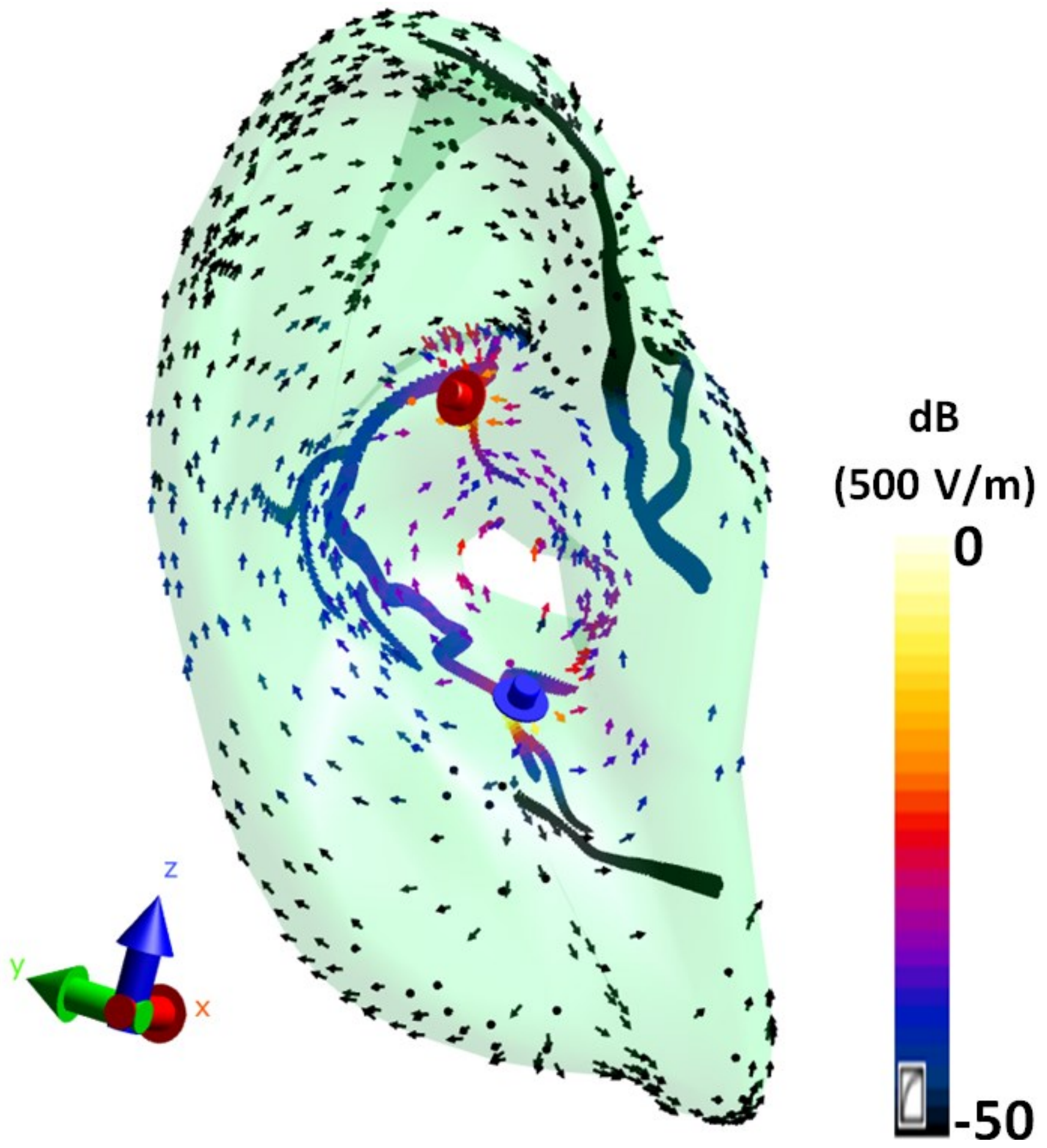

Figure 4 

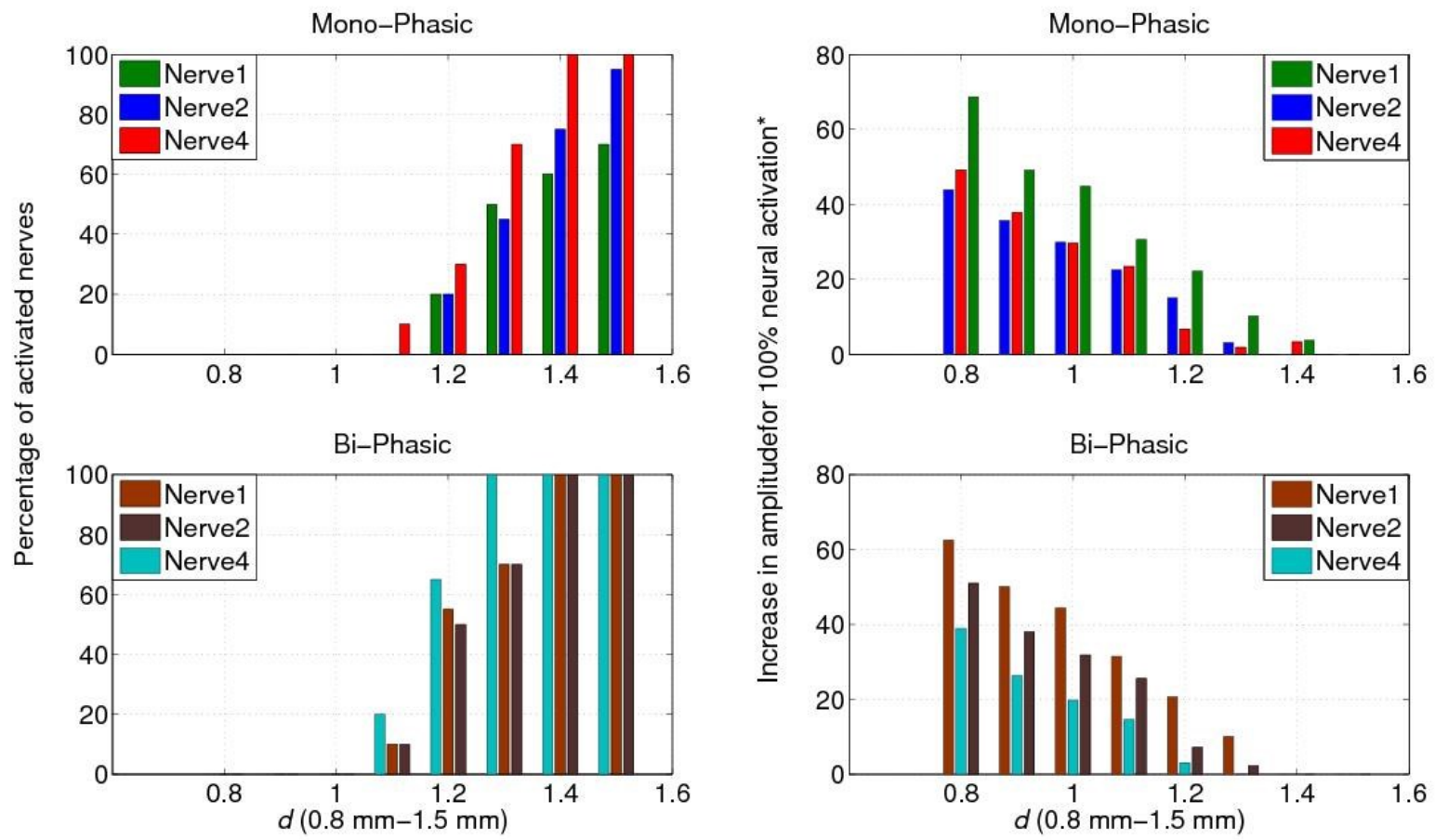

Figure 5 
E-field $(\mathrm{V} / \mathrm{m})$ along nerves for cathodic stimulation
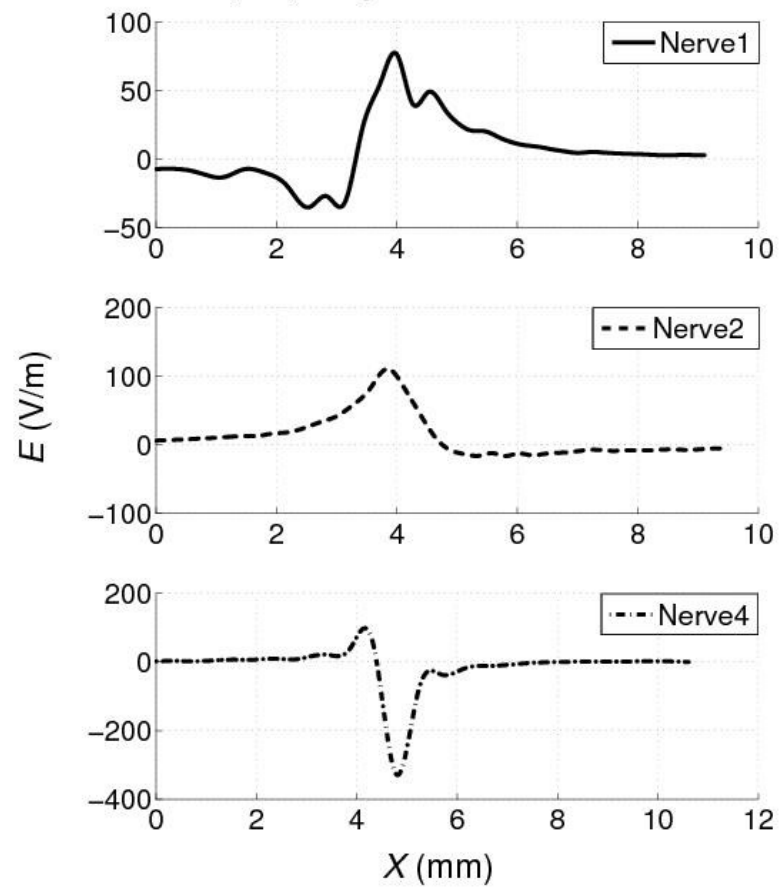

Normalized 1st derivative of the $E$-field along nerves
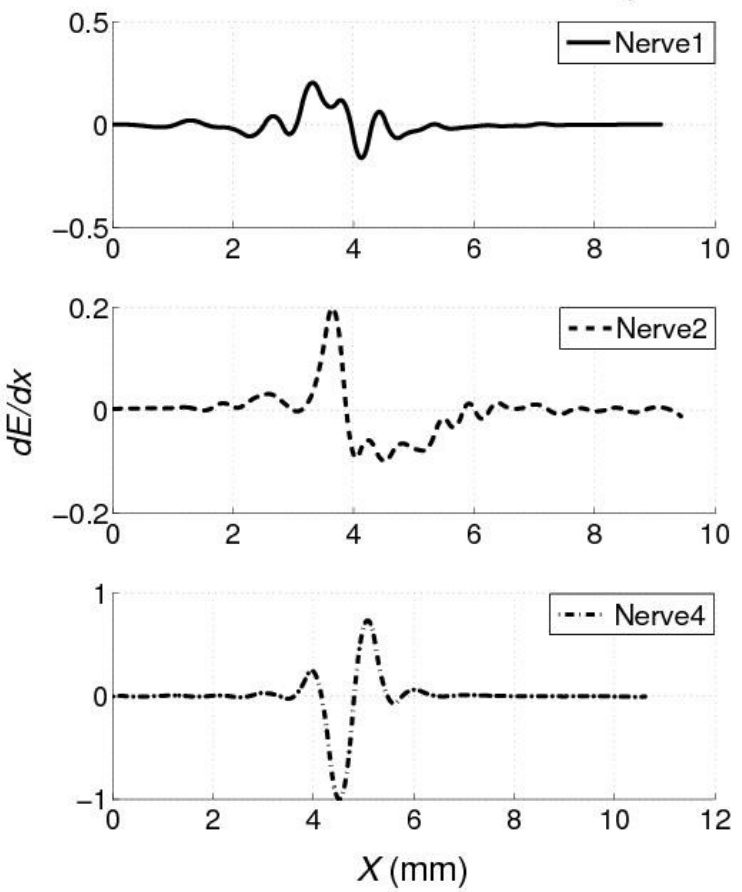

Figure 6 


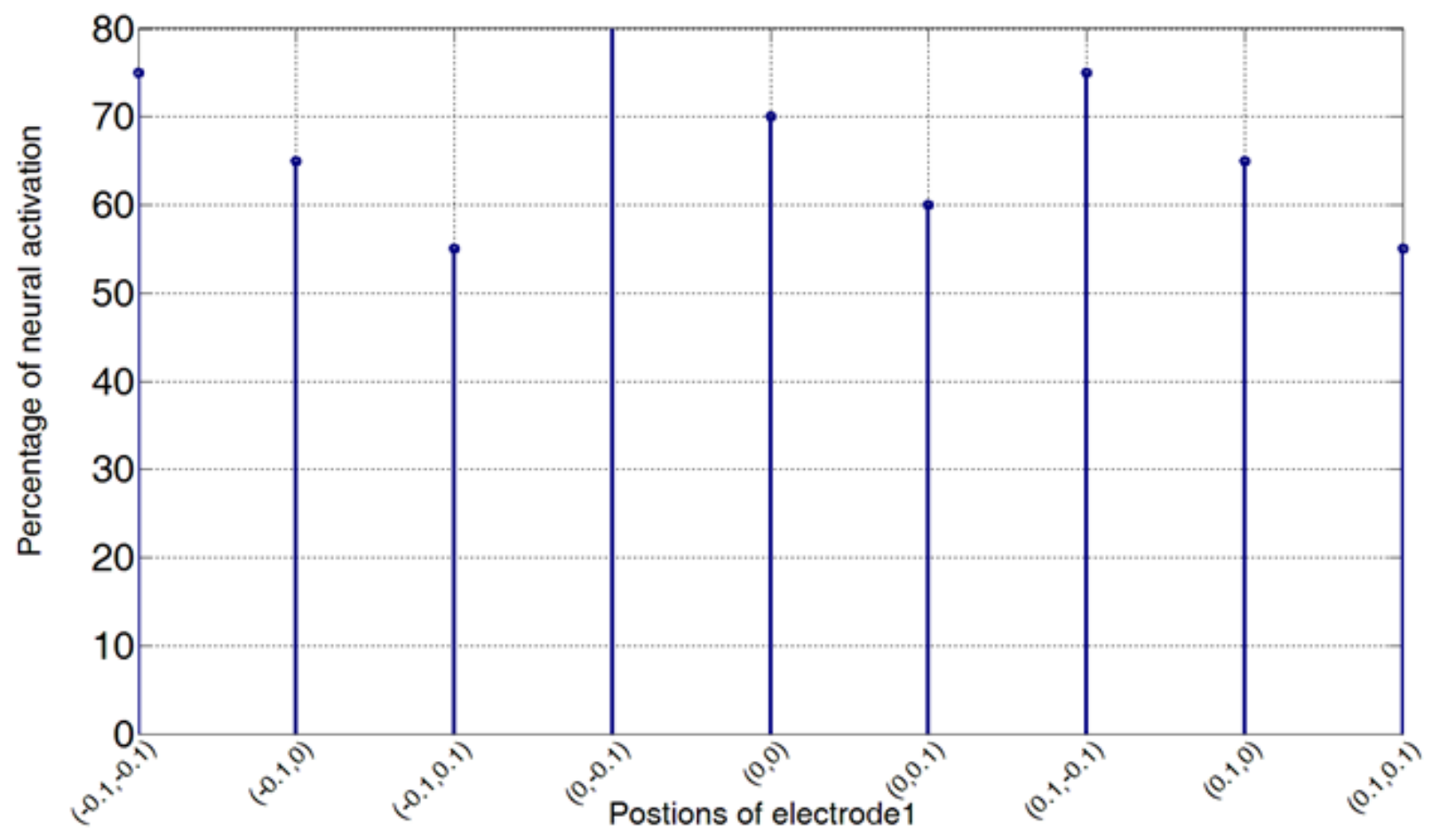

a

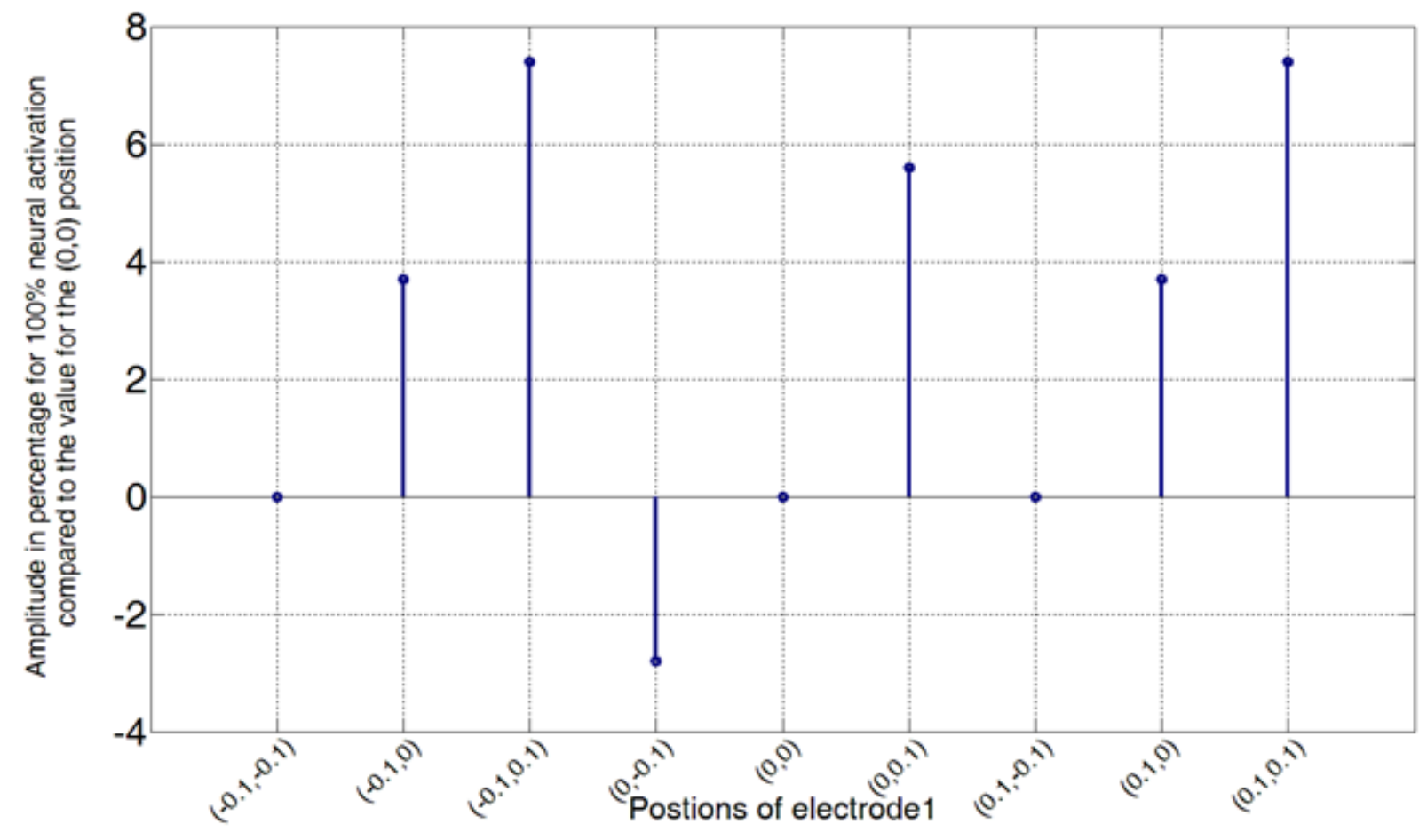

b

Figure 7 